University of Nebraska - Lincoln

DigitalCommons@University of Nebraska - Lincoln

Gerard Harbison Publications

Published Research - Department of Chemistry

May 2005

\title{
Orientation of single crystals using linear approximations to NMR transits
}

Young-Sik Kye

University of Nebraska - Lincoln

Xingang Zhao

University of Nebraska - Lincoln

Gerard S. Harbison

University of Nebraska - Lincoln, gharbison1@unl.edu

Follow this and additional works at: https://digitalcommons.unl.edu/chemistryharbison

Part of the Chemistry Commons

Kye, Young-Sik ; Zhao, Xingang; and Harbison, Gerard S., "Orientation of single crystals using linear approximations to NMR transits" (2005). Gerard Harbison Publications. 4.

https://digitalcommons.unl.edu/chemistryharbison/4

This Article is brought to you for free and open access by the Published Research - Department of Chemistry at DigitalCommons@University of Nebraska - Lincoln. It has been accepted for inclusion in Gerard Harbison Publications by an authorized administrator of DigitalCommons@University of Nebraska - Lincoln. 
Published in Journal of Magnetic Resonance 174:1 (May 2005), pp. 54-59. doi:10.1016/j.jmr.2005.01.013 Copyright (C) 2005 Elsevier Inc. Used by permission. http://www.sciencedirect.com/science/journal/10907807

Submitted November 19, 2004; revised January 17, 2005; published online February 11, 2005.

\title{
Orientation of single crystals using linear approximations to NMR transits
}

\author{
Young-Sik Kye, Xingang Zhao, and Gerard S. Harbison* \\ Department of Chemistry, University of Nebraska-Lincoln, Lincoln, NE 68588-0304, USA \\ *Corresponding author. Email: gerry@setanta.unl.edu
}

\begin{abstract}
We have derived analytical expressions for determining the orientation of high-symmetry single crystals from line-crossings in a single rotation plot. We demonstrate the utility of the method using the strontium-87 resonance in strontium nitrate. Employing our new method, which we call orientation of single crystals using linear approximations to NMR transits (OSCULANT), in combination with fourth-order perturbation theory, we obtain a highly accurate value for the quadrupole coupling constant, and an estimate for the chemical shielding anisotropy.
\end{abstract}

Keywords: Single-crystal NMR, Solid-state NMR, Perturbation theory, ${ }^{87} \mathrm{Sr}$, Chemical shielding anistropy, Quadrupolar nucleus

\section{Introduction}

The most general method for determining magnitudes and orientations of internal NMR interactions uses single crystals to give well-resolved signals, whose orientation dependence can be analyzed to determine the secular part of, or higher-order perturbation contributions to, the nuclear spin Hamiltonian. In the standard method [1] and [2] the crystal is rotated in small increments about three successive, mutually orthogonal axes. Each rotation gives (for a secular, second rank interaction) two diagonal and one off-diagonal element of the Hamiltonian; three rotations thus provide, with some redundancy, all of the elements of the (necessarily symmetric) secular tensor. While more intellectually exciting methods have been proposed [3] using combinations of sample-hopping and multidimensional NMR, single crystal methods remain the mainstay of full tensor determination.

The crystal orientation for each of these three rotation plots is ordinarily obtained by X-ray diffraction or opti- cal goniometry. Both methods require additional effort and equipment. Moreover, fixing the relative orientation of Xray and NMR goniometers, on the one hand, and the orthogonality of the three rotation axes, on the other, remains the principal source of systematic error in single crystal studies. While intelligent design and careful machining [4] can minimize these errors, they cannot eliminate them.

In most crystalline systems, however, there are typically more than one chemically equivalent, magnetically distinct molecular species in the unit cell. The symmetry elements that generate this multiplicity are often regarded as a nuisance, since there is no general way to assign a particular signal to a given molecule. However, it is possible to use the symmetry properties of the unit cell itself, reflected in the NMR rotation plot, to provide absolute orientational information about the crystal in the frame of reference of the goniometer. This is only possible if there exist a multiplicity of NMR-active symmetry operations in the crystal. The most common such operation is the $2_{1}$ screw operation, which (since NMR is oblivious to translations) is 
equivalent in NMR to a $C_{2}$ rotation about a crystallographic axis. Most monoclinic cells have a single such axis, which is insufficient fully to orient the crystal. However, many cells of orthorhombic, tetragonal or cubic symmetry have three mutually orthogonal $2_{1}$ operations. In these cases, it is possible to determine the absolute orientation of the crystallographic $a, b$, and $c$ axes, albeit usually not to distinguish between them (see, however, [5]).

Exploitation of such symmetry has until now been done in a fairly haphazard fashion. In early work [6], deviations of rotation plots from predicted patterns were used to correct small errors in crystal orientation. More recently, we determined [7] the unit cell orientation in an orthorhombic Pbca crystal by iterative fitting to computed rotation plots. However, in the latter case the approximate crystal orientation was determined by optical goniometry, to generate computed rotation plots close enough to experiment to begin an iterative least squares fit.

In this paper, we present a simple method, which we call orientation of single crystals using linear approximation to NMR transits (OSCULANT) to determine the orientation of the crystallographic axis system in the goniometer frame within a single rotation plot. The method works for any NMR Hamiltonian of even rank, and is therefore independent of the nature of the interaction or the crystal (so long as it contains the requisite number of symmetry operations). We will show that it gives excellent results from little more than visual inspection of the rotation plot, and presents a compelling argument for using crystals of high symmetry to determine NMR benchmark information. We demonstrate the method using a single set of rotation plot data for the ${ }^{87} \mathrm{Sr}$ resonances of $\mathrm{Sr}\left(\mathrm{NO}_{3}\right)_{2}$, using fourth-order perturbation theory to calculate the line positions, and then extract highly accurate values for the quadrupole coupling constant and the first estimate of a ${ }^{87} \mathrm{Sr}$ chemical shift anisotropy.

\section{Methods}

A single crystal of $\mathrm{Sr}\left(\mathrm{NO}_{3}\right)_{2}$ of approximate dimensions $0.8 \mathrm{~mm} \times 0.5 \mathrm{~mm} \times 0.3 \mathrm{~mm}$ (Aldrich Chemical, St. Louis, MO, USA) was grown by slow evaporation from aqueous solution at $26^{\circ} \pm 2^{\circ}$. While at this temperature it is possible that either the anhydrous form or the dihydrate might crystallize, in our hands all of the crystals observed were cubic and therefore the anhydrous polymorph. The crystal was mounted in a singly tuned goniometer probe at a magnetic field of $7.187 \mathrm{~T}$ with a coil diameter of approximately $1.2 \mathrm{~cm}$. All spectra were referenced to the ${ }^{87} \mathrm{Sr}$ signal from a freshly opened sample of $\mathrm{SrO}$ (Aldrich), which fell at $13.260013 \mathrm{MHz}$; the measured $90^{\circ}$ pulse on this sample was $7 \mu \mathrm{s}$; however, a small flip angle $1 \mu$ s pulse was used

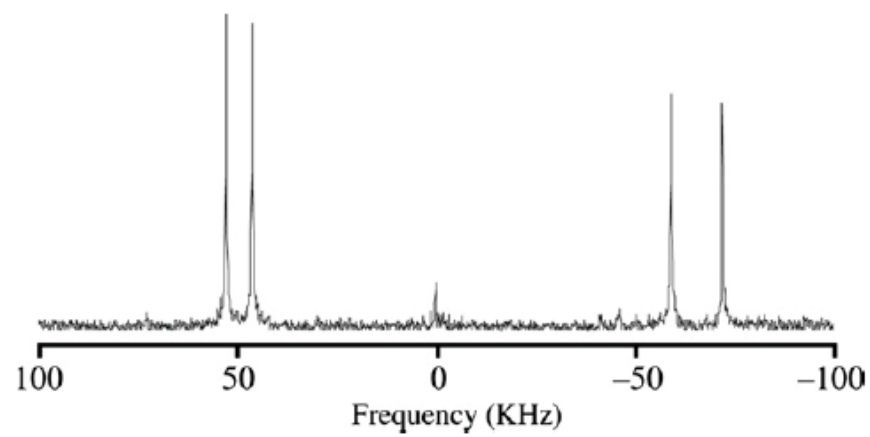

Figure 1. ${ }^{87} \mathrm{Sr} \mathrm{NMR}$ of a single crystal of strontium nitrate, centered at $13.246 \mathrm{MHz}$.

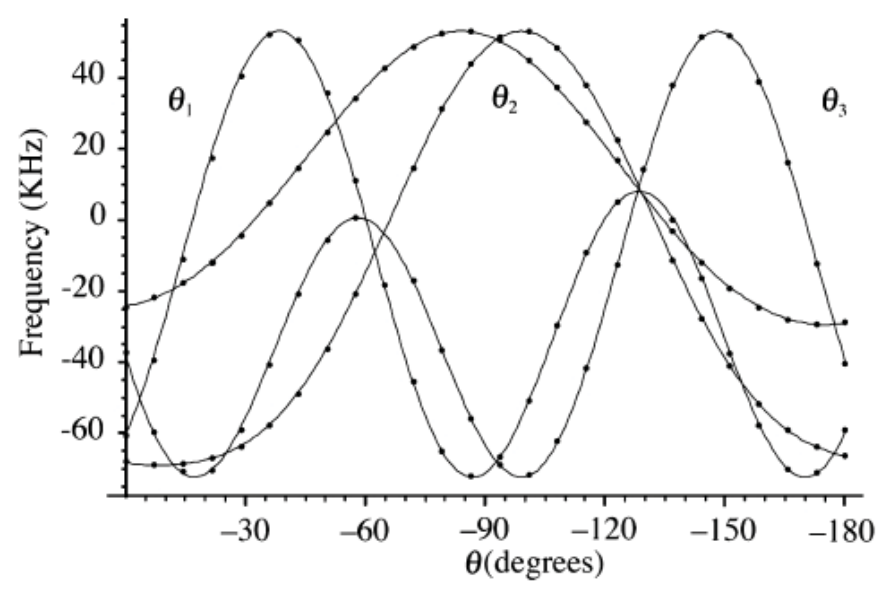

Figure 2. Single crystal rotation plot for strontium nitrate, showing the three transits.

to excite the central transition of $\operatorname{Sr}\left(\mathrm{NO}_{3}\right)_{2}$. For each spectrum typically 15000 transients were signal-averaged with a relaxation delay of $0.5 \mathrm{~s}$; a typical spectrum is shown in Fig. 1. The sample temperature during acquisition was $27 \pm 1{ }^{\circ} \mathrm{C}$. Data were collected in $7.2^{\circ}$ increments for a full $180^{\circ}$ rotation about the goniometer axis. The frequencies of the four identified strontium central transition resonances were obtained by a three-point fit to the peaks, and are plotted in Fig. 2. Note that the sign of the rotation in our NMR probe is negative, and therefore the data are plotted from $0^{\circ}$ to $-180^{\circ}$. The spectrum in Fig. 1 corresponds to $\theta=-86.8^{\circ}$ in the rotation plot.

\subsection{Frames and conventions}

Consider any interaction that can be described by a coupling tensor or combination of coupling tensors. We start with the principal axis frame (PAF) in which the tensor is diagonal. The transformation from PAF to crystallographic frame (XF), which in a crystal of orthorhombic or higher symmetry is defined by the crystallographic $a b c$ axes, is accomplished by the rotation matrix $R_{\mathrm{C}}\left(\alpha_{\mathrm{C}}, \beta_{\mathrm{C}}, \gamma_{\mathrm{C}}\right)$ : 


$$
\begin{aligned}
R_{C}= & R_{z}\left(\alpha_{C}\right) R_{y}\left(\beta_{C}\right) R_{z}\left(\gamma_{C}\right) \\
= & \left(\begin{array}{ccc}
\cos \alpha_{C} & -\sin \alpha_{C} & 0 \\
\sin \alpha_{C} & \cos \alpha_{C} & 0 \\
0 & 0 & 1
\end{array}\right)\left(\begin{array}{ccc}
\cos \beta_{C} & 0 & \sin \beta_{C} \\
0 & 1 & 0 \\
-\sin \beta_{C} & 0 & \cos \beta_{C}
\end{array}\right) \\
& \times\left(\begin{array}{ccc}
\cos \gamma_{C} & -\sin \gamma_{C} & 0 \\
\sin \gamma_{C} & \cos \gamma_{C} & 0 \\
0 & 0 & 1
\end{array}\right) .
\end{aligned}
$$

The four symmetry-related molecules in the unit cell are obtained from this single molecule by the rotation matrices $R_{S, 0}, R_{S, \mathrm{x}}, R_{S, y}$, and $R_{S, z}$ :

$$
\begin{aligned}
& R_{S, 0}=\left(\begin{array}{ccc}
1 & 0 & 0 \\
0 & 1 & 0 \\
0 & 0 & 1
\end{array}\right) ; \quad R_{S, x}=\left(\begin{array}{ccc}
1 & 0 & 0 \\
0 & -1 & 0 \\
0 & 0 & -1
\end{array}\right) ; \\
& R_{S, y}=\left(\begin{array}{ccc}
-1 & 0 & 0 \\
0 & 1 & 0 \\
0 & 0 & -1
\end{array}\right) ; \quad R_{S, z}=\left(\begin{array}{ccc}
-1 & 0 & 0 \\
0 & -1 & 0 \\
0 & 0 & 1
\end{array}\right) .
\end{aligned}
$$

Transformation from this symmetry frame (SF) to the goniometer frame (GF) is accomplished by $R_{G}\left(\alpha_{G}, \beta_{G}, \gamma_{G}\right)$ :

$$
\begin{aligned}
& R_{G}\left(\alpha_{G}, \beta_{G}, \gamma_{G}\right): \\
& R_{G}=R_{z}\left(\alpha_{G}\right) R_{y}\left(\beta_{G}\right) R_{z}\left(\gamma_{G}\right) \\
&=\left(\begin{array}{ccc}
\cos \alpha_{G} & -\sin \alpha_{G} & 0 \\
\sin \alpha_{G} & \cos \alpha_{G} & 0 \\
0 & 0 & 1
\end{array}\right)\left(\begin{array}{ccc}
\cos \beta_{G} & 0 & \sin \beta_{G} \\
0 & 1 & 0 \\
-\sin \beta_{G} & 0 & \cos \beta_{G}
\end{array}\right) \\
& \times\left(\begin{array}{ccc}
\cos \gamma_{G} & -\sin \gamma_{G} & 0 \\
\sin \gamma_{G} & \cos \gamma_{G} & 0 \\
0 & 0 & 1
\end{array}\right)
\end{aligned}
$$

and finally, rotation of the sample in the goniometer, by an angle $\theta$, to the laboratory frame, to generate the actual rotation plot, about an axis which for historical reasons is defined as $x$, is accomplished by

$$
R_{L}=R_{x}(\theta)=\left(\begin{array}{ccc}
1 & 0 & 0 \\
0 & \cos \theta & -\sin \theta \\
0 & \sin \theta & \cos \theta
\end{array}\right) .
$$

The overall transformation from PAF to LF is the product:

$R(\alpha, \beta, \gamma)=R_{C}\left(\alpha_{C}, \beta_{C}, \gamma_{C}\right) R_{S(0, x, y, z)} R_{G}\left(\alpha_{G}, \beta_{G}, \gamma_{G}\right) R_{L}(\theta)$.

And a tensor $T$ in the lab frame is related to its diagonal form $\Lambda_{\mathrm{T}}$ by:

$T=R^{-1}(\alpha, \beta, \gamma) \Lambda_{T} R(\alpha, \beta, \gamma)$.

\subsection{Transits}

Consider two molecules related by a $\pi$ rotation about the $y$ axis of the laboratory frame. If a second-rank symmetric coupling tensor relates the two molecules, the tensor $T$ and $T^{\prime}$ will be identical except in the sign of the $x z$ element, which will be opposite in sign in the two tensors. This element does not contribute to the NMR frequency to first order in perturbation theory, and to higher orders it enters only as even powers. This, the contribution to the NMR frequency of a second rank coupling tensor will be unaffected by a $\pi_{y}$ rotation; and similar arguments can be made for a $\pi_{x}$ rotation.

If the crystal containing these two molecules mounted in a random orientation in a goniometer, and is rotated orthogonal to $\mathbf{z}$, it is infinitely improbable that any of the crystallographic axes will by accident ever lie exactly parallel to $\mathbf{z}$; however, each of these three axes must lie perpendicular to $\mathbf{z}$ twice in any $2 \pi$ rotation (Fig. 3). At the instant in the rotation where either $a, b$, or $c$ axis lies in the $x y$ plane, the NMR frequencies of the molecules related by a $\pi$ rotation about the relevant crystallographic axis become twofold degenerate. In a rotation plot, this is visible as a point where all of the traces of individual NMR line cross pairwise. We label the $a, b$, or $c$ axis transit points as $\theta_{1}, \theta_{2}$, and $\theta_{3}$.

In the present study, we rotated a crystal of $\mathrm{Sr}\left(\mathrm{NO}_{3}\right)_{2}$ about an axis perpendicular to the field, and labeled as $x$, and plotted the frequency of the central transition versus $\theta$. The rotation plot is shown in Fig. 2. The three transits are indicated. Of considerable help in assigning these transits, and in distinguishing between symmetry-related transits and accidental degeneracies, is the knowledge that each curve transits each other exactly once per $\pi$ rotation, and transits of both pairs of rotation plots must coincide exactly. Thus, accidental degeneracies in the plot in the region of $-60^{\circ}$ degrees can be discarded because the exact crossing points do not match. We find that accuracy of a few degrees in estimating the transit angle can be achieved by inspection, while linear intrapolation between the nearest frequencies generally gives an accuracy of better than a degree.

In the $\mathrm{CF}$, the $a$ axis obviously has coordinates $(1,0,0)$. In the lab frame, it has coordinates

$$
a_{L}=R_{L}^{-1}(\theta) R_{G}^{-1}\left(\alpha_{G}, \beta_{G}, \gamma_{G}\right)\left(\begin{array}{l}
1 \\
0 \\
0
\end{array}\right) \text {. }
$$

At $\theta=\theta_{1}$, the $z$ component of $a_{L}, a_{L, z}=0$ (since $a$ now lies in the $x y$ plane). This puts a constraint on the three Euler angles $\alpha_{G}, \beta_{G}$, and $\gamma_{G}$. A second constraint is obtained at $\theta=\theta_{2}$ where $b_{L, z}=0$, and a third at $\theta=\theta_{3}$ where $c_{L, z}=0$. Inverting this set of three equations in $\alpha_{G}, \beta_{G}$, and $\gamma_{G}$ in terms of $\theta_{1}, \theta_{2}$, and $\theta_{3}$ involves a considerable amount of algebra, and so we will give only the results.

$\cos ^{2} \beta_{G}=\frac{1+\cot \theta_{2} \cot \theta_{3}}{\left(\cot \theta_{1}-\cot \theta_{2}\right)\left(\cot \theta_{1}-\cot \theta_{3}\right)}$, 
A

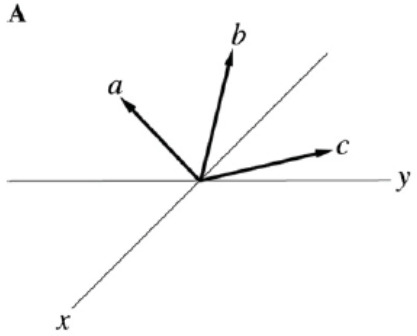

D

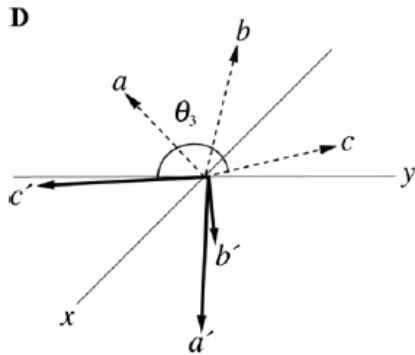

B

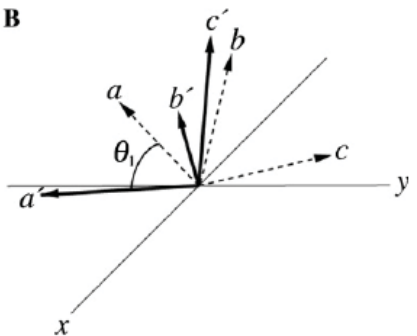

C

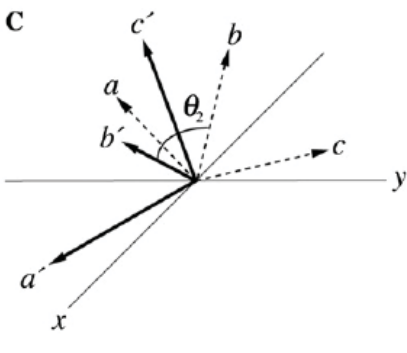

Figure 3. A set of orthogonal crystal axes represented in the lab frame (A) with no rotation, and (B-D) rotated about $x$, by (B) $\theta_{1}$, so $a^{\prime}$ lies in the $x y$ plane (C) $\theta_{2}$, so $b^{\prime}$ lies in the $x y$ plane (D) $\theta_{3}$, so $c^{\prime}$ lies in the $x y$ plane.

$$
\begin{aligned}
& \sin ^{2} \gamma_{G}=\frac{\cot ^{2} \theta_{1}\left(1+\cot \theta_{2} \cot \theta_{3}\right)}{\cot ^{2} \theta_{1}+\cot \theta_{1}\left(\cot \theta_{2}+\cot \theta_{3}\right)-1}, \\
& \sin ^{2} 2 \alpha_{G} \\
& =\frac{4\left(\cot \theta_{1}-\cot \theta_{2}\right)\left(1+\cot \theta_{1} \cot \theta_{2}\right)\left(\cot \theta_{1}-\cot \theta_{3}\right)\left(1+\cot \theta_{1} \cot \theta_{3}\right)}{\left(\cot \theta_{2}-\cot \theta_{3}\right)^{2}\left(1-\cot ^{2} \theta_{1}+\cot \theta_{1}\left(\cot \theta_{2}+\cot \theta_{3}\right)\right)^{2}} .
\end{aligned}
$$

It is immediately apparent that these equations uniquely define $\beta_{G}$ and $\gamma_{G}$ only over the range 0 to $\pi / 2$, and $\alpha_{G}$ only over the range $0<\alpha_{G}<\pi / 4$. However, the expressions for the three cotangents allow these ambiguities to be resolved:

$$
\begin{aligned}
& \sin \gamma_{G} \tan \beta_{G}=-\cot \theta_{1}, \\
& \cot \theta_{2}=\frac{\sin \alpha_{G} \cos \beta_{G} \sin \gamma_{G}-\cos \alpha_{G} \cos \gamma_{G}}{\sin \alpha_{G} \sin \beta_{G}}, \\
& \cot \theta_{3}=\frac{\cos \alpha_{G} \cos \beta_{G} \sin \gamma_{G}+\sin \alpha_{G} \cos \gamma_{G}}{\cos \alpha_{G} \sin \beta_{G}} .
\end{aligned}
$$

There are six possible ways to label the three transits as $\theta_{1}$, $\theta_{2}$, and $\theta_{3}$; and six ways to label the crystallographic axes $a, b$, and $c$ (albeit three of the possible labelings have opposite-handed coordinate systems).

\subsection{Hamiltonian}

By symmetry, the electric field gradient (EFG) tensor at the strontium site in $\operatorname{Sr}\left(\mathrm{NO}_{3}\right)_{2}$ must be axially symmetric, and directed along 111 or symmetry-equivalent directions. In the principal axis frame, whose unique axis coincides with the 111 axis, it is therefore given by

$$
\mathbf{V}=\left(\begin{array}{ccc}
-V_{z z} / 2 & 0 & 0 \\
0 & -V_{z z} / 2 & 0 \\
0 & 0 & V_{z z}
\end{array}\right)
$$

In the PAS frame, the Hamiltonian for the electric quadrupole coupling is:

$$
\begin{aligned}
\mathbf{H}_{Q} & =\left(\frac{e Q}{h(2 I(2 I-1))}\right) \mathbf{I} . \mathbf{V} . \mathbf{I} \\
& =\left(\frac{e Q V_{z z}}{h(4 I(2 I-1))}\right)\left(3 I_{z, P A S}^{2}-\mathbf{I . I}\right),
\end{aligned}
$$

where $\left(e Q V_{z z}\right) / h$ is the so-called quadrupole coupling constant. The angular momentum vector may then be transformed into the laboratory frame:

$I_{z, \mathrm{PAS}}=I_{z} \cos \beta+I_{x} \sin \beta \cos \gamma+I_{y} \sin \beta \sin \gamma$.

As is conventional, the laboratory frame carries no frame designation. The full laboratory Hamiltonian is then

$$
\mathbf{H}=\mathbf{H}_{Z}+\mathbf{H}_{Q}+\mathbf{H}_{\sigma}=\gamma B_{0}(1-\sigma) I_{z}+\mathbf{H}_{Q} .
$$

Assuming the chemical shift to be entirely secular, and treating the quadrupole interaction as a perturbation, we obtain the usual result that the odd-order terms in the perturbation expansion of the quadrupole Hamiltonian for the $1 / 2$ to $-1 / 2$ transition are zero. The second-order perturbation term becomes:

$v_{1 / 2 \rightarrow-1 / 2}^{(2)}=\left(\frac{e Q V_{z z}}{h}\right)^{2} \frac{\left(1-10 \cos ^{2} \beta+9 \cos ^{4} \beta\right)}{384 v_{0}}$,

where $v_{0}=\gamma B_{0}$ is the nuclear Larmor frequency. This expression is identical to that of Volkoff [8] after substituting 


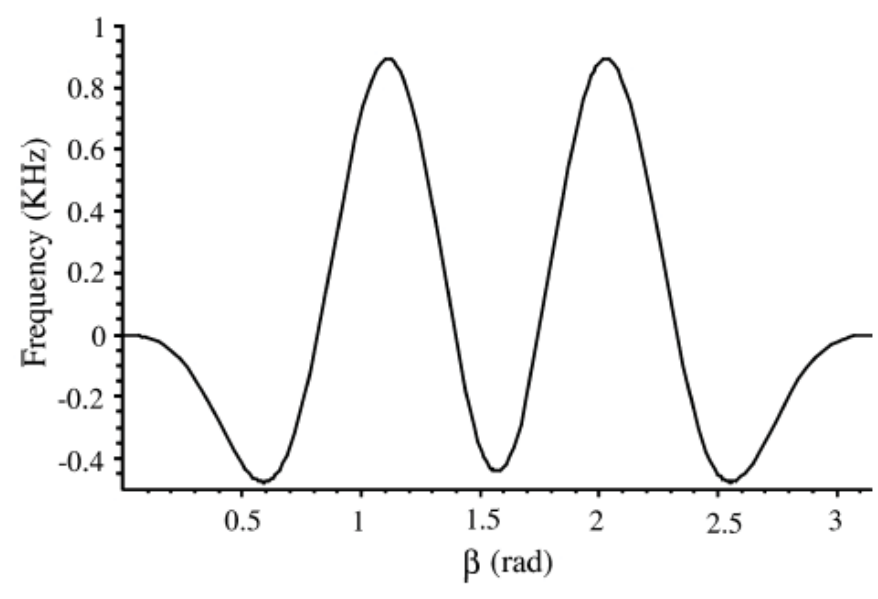

Fig. 4. Computed fourth-order quadrupole coupling for ${ }^{87} \mathrm{Sr}$ in strontium nitrate at $7 \mathrm{~T}$, as a function of the angle $\beta$ between EFG principal axis and the magnetic field.

$\eta=0$. Taking the expansion to fourth order by the method of Löwdin [9], we obtain

$$
\begin{aligned}
v_{-1 / 2 \rightarrow 1 / 2}^{(4)}= & -\left(\frac{e Q V_{z z}}{h}\right)^{4} \\
& \times\left(\frac{17-617 \cos ^{2} \beta+2322 \cos ^{4} \beta-2829 \cos ^{6} \beta+1107 \cos ^{8} \beta}{884736 v_{0}^{3}}\right) .
\end{aligned}
$$

This fourth-order term is plotted in Fig. 4, for a quadrupole coupling constant of $15.16 \mathrm{MHz}$ and Zeeman frequency of 13.2 MHz. The $\cos 6 \beta$ and $\cos 8 \beta$ dependences are clear, and it can be seen that for these values of QCC and Zeeman frequency, the fourth-order contribution is of the order of $1 \mathrm{kHz}$, significantly larger than our final standard error of $560 \mathrm{~Hz}$.

\subsection{Data analysis}

A trial rotation plot was calculated with an estimated QCC of $14.2 \mathrm{MHz}, \beta_{C}=\tan ^{-1} \sqrt{2}$ and $\gamma_{\mathrm{C}}=\pi / 4$ as required by the space-group symmetry, and $\left(\alpha_{G}, \beta_{G}, \gamma_{G}\right)=\left(92.4^{\circ}\right.$, $-82.2^{\circ},-38.4^{\circ}$ ), as determined using Eqs. Figs. (8), (9), $(10),(11),(12)$ and (13) from $\left(\theta_{1}, \theta_{2}, \theta_{3}\right)=\left(-12.5^{\circ},-93^{\circ}\right.$, $\left.-177^{\circ}\right)$. The trial plot was found to lie very close to the experimental data; using it, the four independent rotation plots were assigned, and the fit optimized by least-squares methods, varying the QCC, $\alpha_{G}, \beta_{G}, \gamma_{G}$, and a correction to allow for misalignment of the $x$ axis slightly away from perpendicular to the field. After optimization, the residual was found to contain both $2 \theta$ and higher-order dependences, and therefore, fourth-order corrections and a chemical shift anisotropy term were added (note that only the latter constitutes an additional degree of freedom; the former is merely an improvement in accuracy of the calcula- tions). The fit was reoptimized, giving final values of $\left(\alpha_{G}\right.$, $\left.\beta_{G}, \gamma_{G}\right)=\left(92.4^{\circ},-82.6^{\circ},-40.5^{\circ}\right)$, very close to the initial estimate from the transits. The errors given are $95 \%$ confidence limits, computed for each fit parameter from the total variance and a numerical estimate of the second partial derivative of the variance with respect to each parameter [10]. The goniometer axis misalignment was found to be $0.61^{\circ}$, while the optimized value of the QCC was $15.163 \pm 0.018 \mathrm{MHz}$. The chemical shift with respect to SrO (sigma) was $-428 \pm 9 \mathrm{ppm}$, while the chemical shift anisotropy, $\sigma_{z z}-\sigma_{i}$, was $-23 \pm 28 \mathrm{ppm}$.

\section{Discussion}

The QCC obtained in this work is in fair agreement with the earlier, less precise measurement of $15.4 \pm 0.2 \mathrm{MHz}$, obtained using spin-echo NMR of a strontium nitrate powder [11]. The slightly lower value obtained by us may be due to a difference in the measurement temperature, or it may be a result of the previous authors' use of $\eta$ as a free parameter ( $\eta=0$ by symmetry in this system). The chemical shift anisotropy we obtain, while highly imprecise, is the first estimate we are aware of for ${ }^{87} \mathrm{Sr}$ (with the exception of systems such as $\mathrm{SrO}$ where it is zero by symmetry); it is certainly small and most likely negative, and should be compared with the value of $-35 \mathrm{ppm}$ measured for the more polarizable $\mathrm{Pb}^{2+}$ ion in the isomorphous $\mathrm{Pb}\left(\mathrm{NO}_{3}\right)_{2}$ crystal.

The present example was chosen because it is cubic, and therefore, the issue of assigning the individual $a, b$, and $c$ axes does not arise; in addition, the orientation of the various NMR interactions in the crystallographic frame of reference is fixed by the local point symmetry. It might appear that in the more general case of an orthorhombic crystal with no point symmetry at the NMR nucleus, the inability to assign $a, b$, and $c$ axes poses a problem. In fact, however, this is no more of a problem than if the axes have been assigned by some other means; because, even if the $a, b$, and $c$ axes are known, the four symmetry-related molecules in the unit cell must still be assigned to four sets of NMR resonances. This can either be done using NMR interactions whose direction is known-dipolar splittings or dipolar linewidth - by using atoms which fall at special positions with respect to one of the crystal axes [5], or by comparison of tensor orientations with the local symmetry or with computed tensors. Once this assignment is done, of course, the three axes can also immediately be assigned. The general problem of assignment of chemically equivalent signals in high-symmetry crystals is usually a significant problem only where the chemical shielding anisotropies and local symmetry are low, as, for example, was the case for sucrose [12]. In most cases, in prac- 
tice, assignment is straightforward.

The OSCULANT method breaks down if the rotation plots contain insufficient information to orient the crystal, which can happen if a crystal axis either coincides with, or falls perpendicular to, the goniometer axis. In the former case, all NMR lines become twofold degenerate at all orientations, leaving insufficient information to determine all six tensor elements; in the latter, two transits occur at a single point in the rotation plot, leaving the orientation of the two axes within that plane undetermined. Mounting the crystal close to either of these limiting conditions causes the error in one or more of the fit parameters to become disproportionately large. In practice, we find that they can usually be avoided by making sure that the normals to all crystal faces are neither parallel nor perpendicular to the goniometer axis. Achieving a truly unsymmetrical mounting of the crystal is often surprisingly difficult, since the human brain seems to seek out symmetry even where it is being consciously avoided! Of course, an unfortunate choice is immediately apparent from the rotation plot, where the ideal is three well-spaced transits. And in practice, of course, statistics can be improved by conducting more than one differently mounted rotation, using a simultaneous refinement of tensor magnitudes and orientations while allowing the different crystal orientations to refine independently.

We hope this work convinces the interested reader that, if systematic errors due to crystal mounting can be eliminated by use of the symmetry-derived internal information in a rotation plot, remarkably precise tensors can be obtained, and estimates made of the chemical shielding of higher-spin nuclei even in the presence of very large quadrupolar interactions. This constitutes a strong argument for choosing crystals of orthorhombic or higher symmetry for single-crystal studies, since the disadvantage posed by the assignment problem is outweighed by the increased accuracy of the tensor determination.

\section{Acknowledgment}

This work was funded by a Grant from NIH (R01 GM 065252).

\section{References}

[1] S.D. Gornostansky and C.V. Stager, Nuclear magnetic resonance study of $\mathrm{V}_{2} \mathrm{O}_{5}$, J. Chem. Phys. 46 (1967), pp. 49594962.

[2] M. Mehring, High resolution NMR in solids, Springer, Berlin (1983) (Chapter 2).

[3] M.H. Sherwood, D.W. Alderman and D.M. Grant, Two-dimensional chemical-shift tensor correlation spectroscopy. Multiple-axis sample-reorientation mechanism, J. Magn. Reson. 84 (1989), pp. 466-489.

[4] T. Vosegaard, J. Skibsted, H. Bildsoe and H. Jakobsen, Quadrupole coupling and anisotropic shielding from single-crystal NMR of the central transition for quadrupolar nuclei. ${ }^{87} \mathrm{Rb}$ NMR of $\mathrm{RbClO}_{4}$ and $\mathrm{Rb}_{2} \mathrm{SO}_{4}$, J. Magn. Reson. A 122 (1996), pp. 111-119.

[5] R. Subramanian, N. Govindaswamy, R.A. Santos, S.A. Koch and G.S. Harbison, Single-crystal, solid-state and solution ${ }^{113} \mathrm{Cd}$ and ${ }^{77} \mathrm{Se} \mathrm{NMR}$ and X-ray single crystal study of an [Cd$\mathrm{SeR}_{2} \mathrm{~N}$-donor ${ }_{2}$ ] complex, Inorg. Chem. 37 (1998), pp. 49294933 .

[6] G.S. Harbison, J. Herzfeld and R.G. Griffin, Nitrogen-15 chemical shift tensors in 1-histidine hydrochloride monohydrate, $J$. Am. Chem. Soc. 103 (1981), pp. 4752-4754.

[7] G.S. Harbison, Y.-S. Kye, G.H. Penner, M. Grandin and M. Monette, ${ }^{14} \mathrm{~N}$ Quadrupolar, ${ }^{14} \mathrm{~N}$ and ${ }^{15} \mathrm{~N}$ chemical shift, and ${ }^{14} \mathrm{~N}-{ }^{1} \mathrm{H}$ dipolar tensors of sulfamic acid, J. Phys. Chem. B 106 (2002), pp. 10285-10291.

[8] G.M. Volkoff, Second order nuclear quadrupole effects in single crystals. I. Theoretical, Can. J. Phys. 31 (1953), pp. 820836.

[9] P.-O. Löwdin, Studies in perturbation theory. IX. Connection between various approaches in the recent development-evaluation of upper bounds to energy eigenvalues in Schrödinger's perturbation theory, J. Math. Phys. 6 (1965), pp. 1341-1353.

[10] T. Vosegaard, E. Hald, V. Langer, H.J. Skov, P. Daugaard, H. Bildsøe and H.J. Jakobsen, Improved hardware and software for single-crystal NMR spectroscopy, J. Magn. Reson. 135 (1998), pp. 126-132.

[11] F.H. Larsen, J. Skibsted, H.J. Jakobsen and N.C. Nielsen, Solid-state QCPMG NMR of low- $\gamma$ quadrupolar metal nuclei in natural abundance, J. Am. Chem. Soc. 122 (2000), pp. 70807086.

[12] M.H. Sherwood, D.W. Alderman and D.M. Grant, Assignment of carbon-13 chemical-shift tensors in single-crystal sucrose, $J$. Magn. Reson. 104 (1993), pp. 132-139. 